\title{
Special issue: Advances in landslide understanding
}

\author{
Guest Editors \\ Eduardo E. Alonso and Núria M. Pinyol \\ Universitat Politècnica de Catalunya, Barcelona, Spain
}

